\title{
Fungi in human pathology - are we ready to handle them?
}

\author{
Adrian Man* \\ Department of Microbiology, University of Medicine, Pharmacy, Sciences and Technology of \\ Tîrgu Mureş, Romania; Central laboratory, Mureș Clinical County Hospital, Romania.
}

Received: 25 ${ }^{\text {th }}$ September 2018; Accepted: $1^{\text {st }}$ October 2018; Published: $3^{\text {rd }}$ October 2018

Fungi are ubiquitous organisms and essential components of natural ecosystems, with many positive effects. Beside bacteria, they are natural recyclers on the Earth. Nevertheless, nowadays, together with the high incidence of conditions associated with immune-depression and with the development and implementation of prosthetic devices in humans, the negative effects of fungi gain more and more interest. The imbalance between the presence of fungal pathogenicity factors and the compliance of the host defense systems is the main determining factor for fungal infections outcome, but allergies, mycotoxicoses and food poisonings are also unwanted effects of fungi over the human body.

The regnum of fungi includes a high variety of eukaryotic micro- and macro-organisms, where the number of genera and species can only be approximated. The taxonomy of fungi is on constant change, as the DNA sequencing methods bring new evidence for their classification. The fungi form their own microsystem on Earth, with different behavior, complex interaction between them and the environment, thus making them unpredictable in relation with their toxicity and pathogenicity. The impact of these constant changes reflects on the ecosystem, but not the least, on human pathology (either as toxicoses or infections) (1).

Yeast pathology represents one of the most common fields of research in this large microbiological branch of mycology. Candida spp. are the most well described and studied fungal species, as they are the most common etiological agents of fungal infections. Several comorbidities are known to be associated with yeast infections, where diabetes mellitus, acquired or primary immune-deficiencies, malignancies are only of the few that are constantly described. Usually, some imbalances or presence of some favoring factors will finally lead to the development of fungal infections. Not only the virulence factors, but also the replication rate of yeasts are key factors for the disease. Recent studies that simulated the internal conditions of a diabetic patient, showed that the replication rate of Candida albicans is increased in the presence of glucose, but decreased in the presence of fructose (a common sweetener used by diabetic patients), or that metformin, a common antidiabetic drug,

*Corresponding author: Adrian Man, Department of Microbiology, University of Medicine, Pharmacy, Sciences and Technology of Tîrgu Mureş, Romania; Central laboratory, Mureş Clinical County Hospital, Romania.

E-mail: adrian.man@umftgm.ro 
drastically increases the replication rate and slightly increases the germ-tube formation $(2,3)$ Some hypotheses suggest that yeast colonization of the oral cavity directly influences the tendency of an individual to develop type 2 diabetes, by inducing insulin-resistance (4).

There is a thin line between yeast colonization and infection. The interpretation of laboratory findings has to be performed critically, as some human sites can be colonized with yeasts, without clinical implications. For example, the presence of Candida spp. in sputum can be due to the improper harvesting of the pathological product, being actually o contamination from oral sites, especially if the patient is an elder with dental prosthesis. Instead, growth of Aspergillus spp. from sputum can have clinical significance, in the context of a pulmonary aspergillosis. Development and progression of yeast infections in colonized (oral or vaginal) mucosa and to adjacent sites is possible, in the context of different favoring factors such as the use of antibiotics, alcohol intake, smoking or radiotherapy (5).

Many studies are required in order to bring details on the epidemiology of fungal infections. These studies have to go beyond the classic approach, where patient data were correlated with laboratory data. For example, further correlations between genotypes and pathogenicity factors are bringing more evidence on the interrelation between host and microorganism. An epidemiological study published on this issue follows these factors that contribute to the pathogenic potential of C. albicans (6), and it was found that the levels of enzymatic activity of $C$. albicans is a strain-dependent variable, and that not all strains were capable to produce biofilm. Such details are important to be considered when dealing with Candida infections, remembering that even if the identified species is the same from different patients/samples, they can also be totally different in terms of pathogenicity and chemo-resistance.
Beside the common fungal infections which are intensively studied, another type of pathology is associated with fungi: the mycotoxicoses. These are non-invasive fungal diseases, which are developed usually due to ingestion of fungal secondary metabolites, called mycotoxins, such as aflatoxin, zearalenone, fumonisins or ochratoxin. Acute effects of mycotoxins are easy to follow in vivo. Weight loss, liver and kidney injury, metabolic alterations, enzymatic impairment, immune-suppression or tumor development are only a few of the effects produced by high doses of mycotoxins $(7,8)$. Instead, the long-term exposure and accumulation of mycotoxins is hard to predict and control. The effects of mycotoxins, though well-described in vitro studies, are difficult to be interpreted in real life, as different pathological conditions may be expressed by multiple factors which can overlap the assimilation of small but continuous amounts of mycotoxins. In addition, the food industry, though governed by food safety legislation, is hard to be properly controlled, so higher amounts of mycotoxins than permitted by law may be present in various products. Local market products, even common spices (9), were found to be full of fungi and mycotoxins.

If the treatment of mycotoxicoses is virtually inexistent, the treatment options of fungal infections are available, but limited to the few antifungal classes: azoles, polyenes, echinocandins, allylamines and nucleoside analogs. The antifungal susceptibility testing methods are more demanding and harder to implement as routine than the antibiotic susceptibility testing, requiring quantitative detection methods (microdilution, automated breakpoint detection methods - e.g. Vitek2 systems). For the detection of antifungal resistance, simple PCR methods are limited, as the resistance to azoles is not encoded by a specific gene (similarly as antibiotic resistance is encoded in many bacteria), but rather by gene mutations or target site upregulations, which are 
detectable only by gene sequencing, qPCR for allele discrimination, RT-PCR for gene expression/miniarrays. The resistance can be induced by suboptimal dose use of azoles, so the injudicious use of antifungal drugs (e.g treatment of a yeast colonization) can have non-desired effects for a long time. Many epidemiological studies assess the antifungal susceptibility of clinical isolates, in order to create a wider view on the fungal resistance phenomenon (10-12). This would bring evidence that can be successfully used for the implementation of an empirical treatment, depending on the pathology, infection site and identified species.

To conclude the initial question, we should be able to handle the fungal infections if we extract and take into account the essential information from the literature, following the epidemiology, antifungal resistance patterns, clinical status of the patient or favoring factors for infections. We should not forget about the particularities and individual characteristics of fungal strains when dealing with treatment failure.

\section{Conflict of interest}

None to declare

\section{References}

1. Steenkamp ET, Wingfield MJ, McTaggart AR, Wingfield BD. Fungal species and their boundaries matter - Definitions, mechanisms and practical implications. Fungal Biol Rev. 2018 Mar 1;32(2):104-16. DOI: 10.1016/j.fbr.2017.11.002

2. Man A, Ciurea CN, Pasaroiu D, Savin A-I, Toma F, Sular F, et al. New perspectives on the nutritional factors influencing growth rate of Candida albicans in diabetics. An in vitro study. Mem Inst Oswaldo Cruz. 2017 Sep;112(9):587-92. DOI: 10.1590/0074-02760170098
3. Ciurea CN, Mare A, Man A. The influence of metformin on Candida albicans growth and germ tube formation. 11th edition of the ASKLEPIOS International Congress for Students and Young Physicians, Sibiu, Romania. Abstract book ISSN 1843-0406. 2018:197.

4. Monea A, Santacroce L, Marrelli M, Man A. Oral candidiasis and inflammatory response; A potential synergic contribution to the onset of Type-2 Diabetes Mellitus. Australasian Med J. 2017;10(6):550-556. DOI: 10.21767/AMJ.2017.3053

5. Petrovan C, Bereczki-Temistocle DL, Man A. Candida in Oral and Maxillofacial Pathology: Clinical Findings and Risk Factors. J Interdiscip Med. 2017 Mar 1;2(s1):36-42. DOI: 10.1515/jim-2017-0013

6. Rosca I, Bostanaru AC, Minea B, Nastasa V, Gherghel I, Panzaru CV, et al. Candida albicans isolates from Romanian patients. Rev Romana Med Lab. 2018 Jul 6;26(aop). DOI: 10.2478/rrlm-2018-0023

7. Zain ME. Impact of mycotoxins on humans and animals. J Saudi Chem Soc. 2011 Apr 1;15(2):129-44. DOI: $10.1016 /$ j.jscs.2010.06.006

8. Bennett JW, Klich M. Mycotoxins. Clin Microbiol Rev. 2003 Jul;16(3):497-516. DOI: 10.1128/CMR.16.3.497516.2003

9. Man A, Mare A, Toma F, Curticăpean A, Santacroce L. Health Threats from Contamination of Spices Commercialized in Romania: Risks of Fungal and Bacterial Infections. Endocr Metab Immune Disord Drug Targets. 2016;16(3):197-204. DOI: 10.2174/187153031666616 0823145817

10. Agrosoaie R, Streinu-Cercel A, Azoicai D, Bejan C, Dorobat O, Mihai A, et al. Epidemiological data and antifungal susceptibility in invasive fungal infections a Romanian infectious diseases tertiary hospital's experience. Preliminary report. Rev Romana Med Lab. 2017 Aug 21;25(4):345-53. DOI: 10.1515/rrlm-2017-0023

11. Földes A, Bilca D-V, Székely E, Enikő F, Toma F. Involvement of yeast species in fungaemia - an investigation of clinical data. Rev Romana Med Lab. 2008;13(4):49-58.

12. Minea B, Nastasa V, Moraru RF, Kolecka A, Flonta MM, Marincu I, et al. Species distribution and susceptibility profile to fluconazole, voriconazole and MXP4509 of 551 clinical yeast isolates from a Romanian multi-centre study. Eur J Clin Microbiol Infect Dis Off Publ Eur Soc Clin Microbiol. 2015 Feb;34(2):367-83. DOI: $10.1007 /$ s10096-014-2240-6 